Thorax (1946), 1, 128.

\title{
ANOXIA AND THE CENTRAL NERVOUS SYSTEM : AN EXPERIMENTAL AND CLINICAL STUDY
}

\author{
B. G. B. LUCAS
}

London

\section{INTRODUCTION}

The purpose of this paper is $(a)$ to describe the effects of prolonged mild anoxia on the brains of experimental animals, and $(b)$ to record a series of cases showing the effects of anoxia on the human brain. It is not intended to give a detailed account of either the different types of anoxia or the mechanisms whereby these types occur.

The effects of severe cerebral anoxia, causing death in a few hours, are well known, but there is less agreement whether mild cerebral anoxia by itself is dangerous. It has been thought that it was not, and that any brain changes which occurred were temporary in character. People recover from acute asphyxia every day without any apparent after-effects ; and nitrous oxide, whose anaesthetic properties according to Brown and others (1927) are due to anoxic anoxia, is considered to be one of the safest of all anaesthetic agents.

Courville (1938) drew attention to the after-effects of asphyxia and showed that permanent damage to the brain could occur. But his work consisted mainly of the collection of clinical data, and he offered no special evidence to explain why damage occurred in most cases after moderate or mild anoxia, whereas subjects sometimes recovered following an exposure to severe anoxia. The first clue to this discrepancy was found by Thorner and Lewy (1940), who, working with guinea-pigs, found that there was a progressive and accelerated cerebral change with repeated doses of anoxia. They submitted the animals to daily doses of severe asphyxia, by placing them in a chamber filled with nitrogen until respiration ceased. They then removed and resuscitated them. After about nine to twelve exposures the animals were killed and examined. In all cases the brain showed a progressive destruction of nerve cells in proportion to the number of exposures.

Previous to the work of Thorner and Lewy, Dellaporta (1939) described experiments in which he subjected guinea-pigs to low oxygen tensions, by decompression in air to $250 \mathrm{~mm}$. $\mathrm{Hg}$ for periods up to 100 hours. If the animals were 
killed immediately after the decompression, no demonstrable changes in the central nervous system were found, but if the animals died some hours after their exposure to low pressure, then changes in the brain were observed.

A year later Merk (1940) subjected guinea-pigs to periods of low atmospheric pressure $(198 \mathrm{~mm}$. $\mathrm{Hg}$ ) for one hour every day until the animals succumbed. In all cases he demonstrated nerve cell changes in the brain.

The experimental evidence offered by all these authors suggested that repeated or prolonged periods of severe anoxia had a cumulative effect on the brain. It was therefore decided to carry out experiments in which animals were subjected to periods of mild anoxia only. Adult rats were used as experimental animals because they are more easily obtained than guinea-pigs, and because whole brains can be stained and sectioned. One disadvantage, however, is that rats are more capable of withstanding anoxia than larger animals.

\section{EXPERIMENTAL OBSERVATIONS}

The method of obtaining anoxic conditions was as follows. The rats were placed in a gas-tight chamber which was connected to a spirometer with a capacity of 150 litres. The gases were circulated by a pump through soda-lime to remove carbon dioxide, and were kept at a constant temperature by thermostatically controlled air heaters. The gas mixture was analysed every hour, and was kept constant within \pm 0.5 per cent by the occasional addition of small quantities of oxygen.

Two rats were used at each experiment. In most cases the experiment was continued until one rat died. Both were then removed from the chamber. The survivor was killed by decapitation after three days. Whenever possible the brains were removed and fixed immediately after death, but in some experiments the fixation was delayed up to five hours. The staining methods employed were Ehrlich's haematoxylin and chromotrope, iron haematoxylin, and Nissl's.

At the outset continuous exposures to oxygen tensions of 3 to 4 per cent were used. The animals survived for periods of approximately half to one hour. As the oxygen content was raised so the survival time was lengthened, until with 9 per cent oxygen it was approximately three days.

A different method was then employed, in which the animals were subjected to the same degree of anoxia ( 9 per cent oxygen) for periods of seven hours daily, when their survival time was approximately nine to ten days. This compares directly in time (63 to 70 hours) with the rats of the previous group, which were anoxic continuously for three days (72 hours). What is more interesting is that on only four occasions out of fourteen with the two latter groups was it necessary to kill the surviving animal in three days. In all the rest of the experiments the survivor died within three days of being removed from the chamber. In one experiment the lungs were sectioned, and those of the survivor which died two days afterwards were found to be oedematous. 
It has already been stated that rats appear to be more resistant to anoxia than other animals, and in 9 per cent oxygen they appeared to be completely conscious, if a little dazed; during their periods of anoxia they drank fairly freely, but made very little attempt to eat.

Microscopic examination of the brain in the acute experiments revealed very little, but as the anoxia became more prolonged, evidence of damage began to appear. This was similar to that already observed by Gildea and Cobb (1930) and Oster, Toman and Smith (1944), consisting of destruction of nerve cells and loss of Nissl staining properties. All the animals which died outside the chamber in the chronic experiments showed similar damage to those which died when actually anoxic.

Using ordinary staining methods, the best evidence of cell damage was found in the area of the cornu ammonis. Normally two groups of nerve cells are to be found ; a layer of loosely arranged large pyramidal cells which are deeply staining and a layer of tightly packed small round cells. After prolonged anoxia, the large pyramidal cells gradually lose their staining properties and finally almost disappear, whereas the small round cells become more deeply staining and rather more irregular and shrunken in outline. The decrease in staining of the pyramidal cells and the increase in the small round cells were more noticeable in the "surviving" animals, that is, those which did not die during the experiment but were killed or died later (Figs. 1 to 3,5 to 7 and 9 to 11).

Besides signs of damage to nerve cells, there was also a great increase in number of capillaries visible throughout the brain. That this may have been partly due to an actual formation of new capillaries is suggested by the observation that several normal brain sections especially stained for blood vessels failed to show as many capillaries even in the collapsed state as were present dilated in the brains from the experimental animals. However, only part of the increase can have been due to new capillaries, the remainder being a dilation of the existing ones, since the animals which died while anoxic showed a greater increase than those which died later (see Figs. 4, 8, and 12). This increase in blood vessels has also been found by Yaskin and Thorner (1943) in other states of chronic anoxia, particularly carbon monoxide poisoning.

The microscopic changes in the brain found in these experiments are similar to those which have been found in man by Helwig (1937) following severe asphyxia, and by a number of authors including O'Brien and Steegmann (1938), Suggs (1943), Lenahan (1943), and Steegmann (1939) following anaesthesia.

This preliminary report indicates that prolonged mild anoxia, in animals at any rate, can cause severe damage to the brain, and that the effect is cumulative.

\section{Effects of Anoxia on Man: Illustrative Cases}

The following eight cases illustrate the serious effects of anoxia on the central nervous system in man. They have been collected from various sources over a 
period of five years, and only the details relevant to the anoxia are included. Six of these cases were associated with anaesthesia. They are placed in decreasing order of severity, the most severe ones being included to illustrate the extreme clinical and pathological changes that anoxia does produce in man.

CaSe I (Male, aged 19). Obese but otherwise healthy. Tonsillectomy was intended. During induction of anaesthesia with intravenous avertin, after $1.5 \mathrm{c.cm}$. the heart and respiration stopped simultaneously. Immediately, an endotracheal tube was passed and the lungs inflated with oxygen; $5 \mathrm{c.cm}$. of nikethamide was then injected into the right auricle. This produced no response, so direct cardiac massage was started. The delay in starting massage was about three to four minutes. With manual compression of the heart, the blood appeared more oxygenated, and after three minutes the heart started beating again. Cardiac massage was stopped. After two minutes the heart stopped again, and was restarted with cardiac massage. This occurred twice more until, 18 minutes after the heart had originally stopped, it was beating steadily and respiration was normal. The abdominal incision for the cardiac massage was then sewn up and the patient returned to the ward and given oxygen through a B.L.B. mask.

On return to the ward his pulse rate was 90 , blood pressure 100/70 and respiration 20 . Within half an hour moist sounds appeared at both bases, and within an hour the lungs were very oedematous. He was still deeply unconscious, with all reflexes absent and dilated pupils. Four hours later his mental condition was unchanged, but now his lungs were completely waterlogged. He was dyspnoeic, respiration rate was 50 , pulse rate 140 , and temperature $101^{\circ} \mathrm{F}$. His pupils were now slightly contracted but reacted to light. His condition gradually deteriorated and he died nine hours after his heart had originally stopped. His temperature just prior to death was $107^{\circ} \mathrm{F}$.

Post Mortem : Nothing abnormal found except marked congestion and oedema of both lungs. The pial vessels of the brain were congested and the whole brain tissue was softer than normal. No gross evidence of brain destruction was observed. No microscopy was done.

Comment : In this case the cerebral anoxia was extremely severe, the arrest of the circulation being complete for three minutes and partial for a further fifteen. This was enough to destroy fore-brain function, leaving only the respiratory centre. The further anoxia from the pulmonary oedema completed the picture.

CASE II (Male, aged 56). General condition fair. Operated on for maxillary sinusitis. Induction of anaesthesia following omnopon gr. 1/3 and scopolamine gr. 1/150 was with one gramme of pentothal. A small (No. 5) Magill endotracheal tube was then passed and nitrous oxide, oxygen, and ether were given. Because of excess bleeding (due, possibly, to the small tube) chloroform was added. Throughout the operation the patient's colour was dusky and on return to the ward it was still poor; he stopped breathing twice and had to be resuscitated.

After twelve hours he was still unconscious, although he responded to painful stimuli with groans. The Babinski test produced a mass withdrawal of the entire lower limb. $\mathrm{He}$ never recovered from this state and died three days later.

Post Mortem: In the white matter of all areas of the brain there were foci consisting of minute haemorrhages with adjacent areas showing purplish discoloration (Fig. 13). The membranes and vessels appeared normal. Microscopically each lesion was seen to involve both the grey and the white matter, and was characterized by an enormous dilatation of blood vessels. There were patches of degeneration in these lesions, but little or no evidence of cellular infiltration (Fig. 14). 


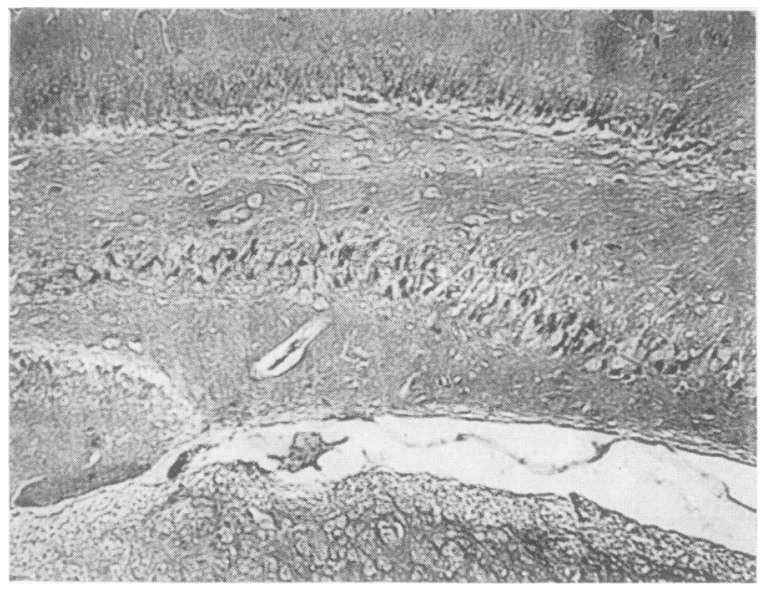

Fig. 1.-Cornu Ammonis $(\times 60)$ of normal rat brain, showing deeply staining pyramidal cells and small nerve cell layer.

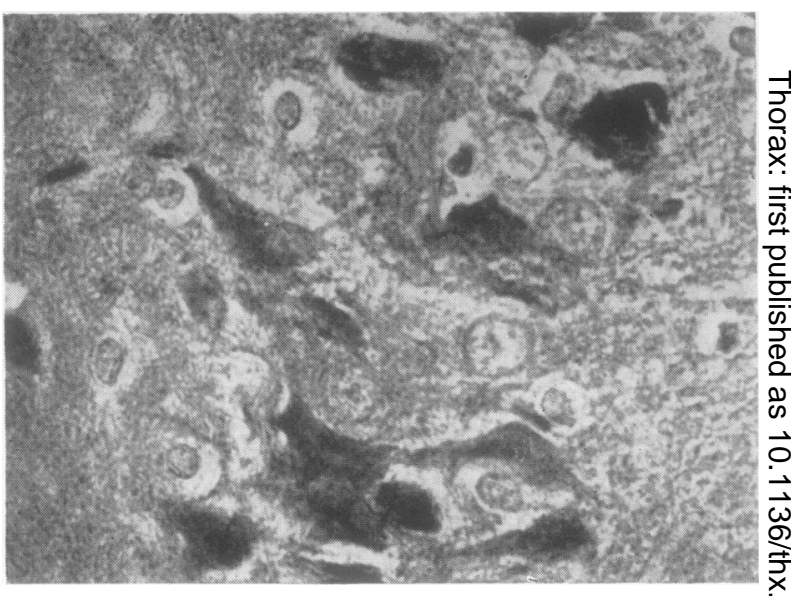

Fig. 2.-Pyramidal cell area of Fig. 1 enlarged $(\times 300)$. Note well staining large pyramidal cells.

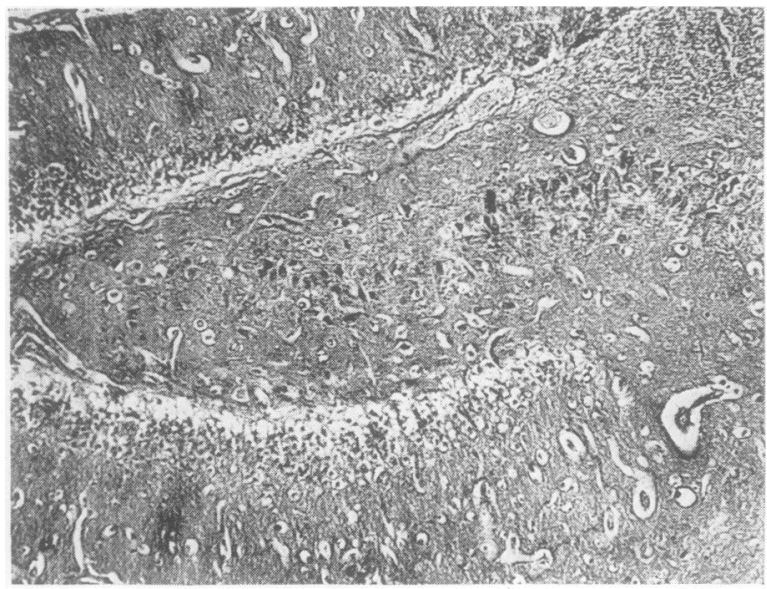

Fig. 5.-Cornu Ammonis $(\times 60)$ of anoxic rat brain, showing partial loss of pyramidal cells. The animal died in the chamber.

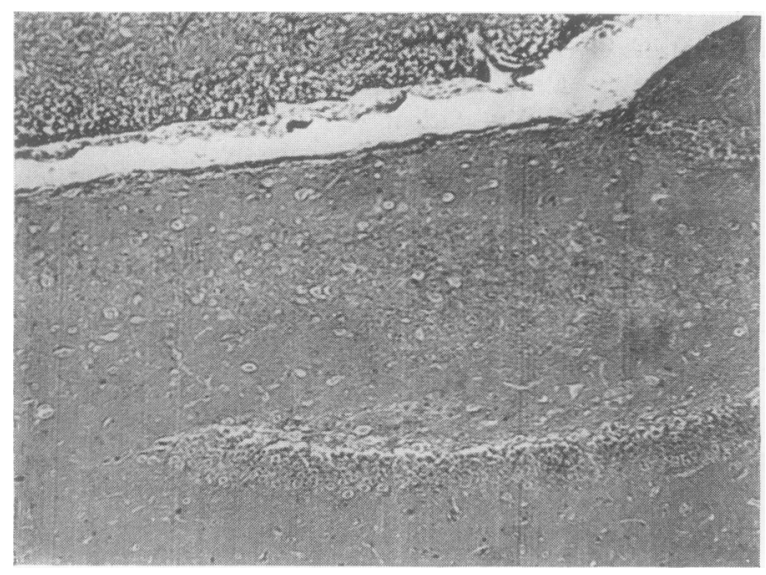

Fig. 9.-Cornu Ammonis $(\times 60)$ of anoxic rat brain The animal died outside the chamber. Note complete loss of pyramidal cells.

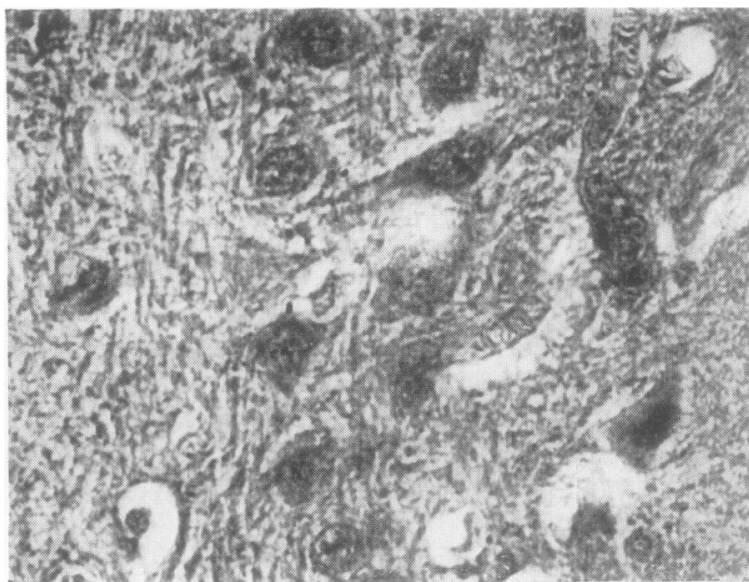

FIG. 6.-Pyramidal cell area of Fig. 5 enlarged $(\times 300)$. Note loss of staining and partial loss of pyramidal cells.

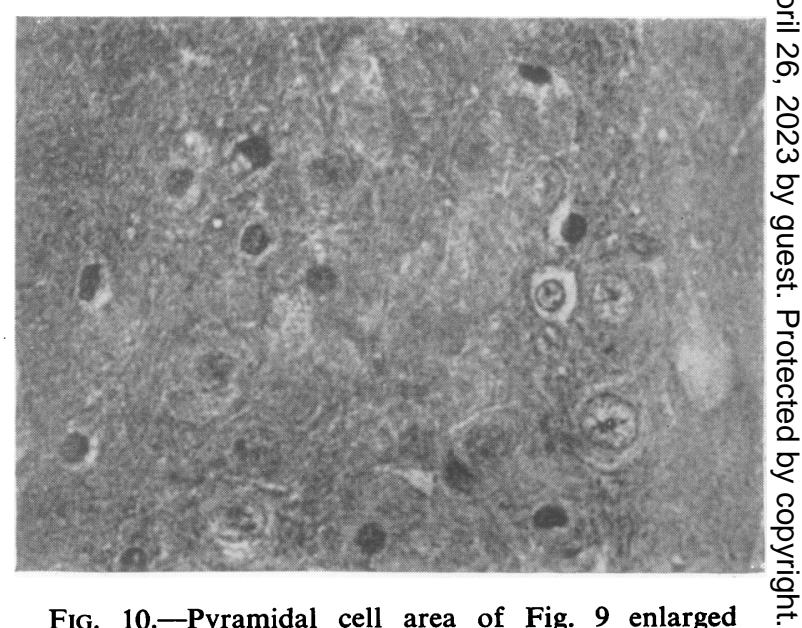

Fig. 10.-Pyramidal cell area of Fig. 9 enlarged $(\times 300)$. Note almost complete disappearance of pyramidal cells. 


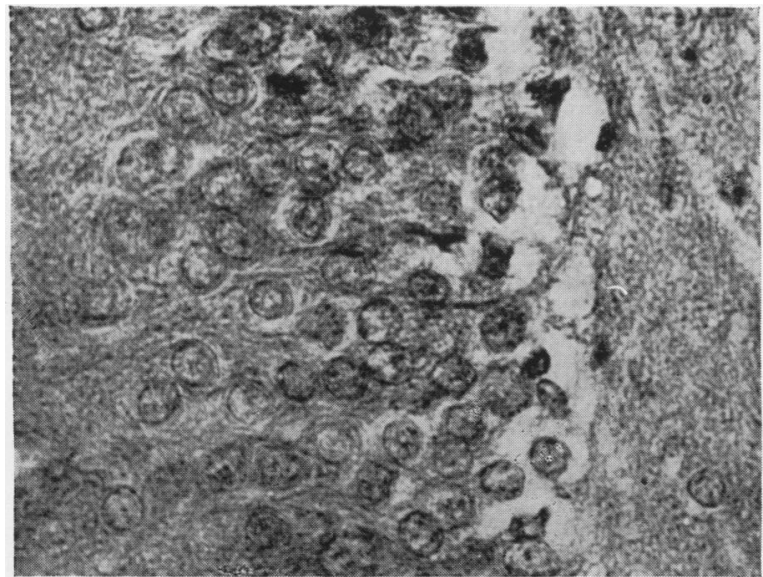

Fig. 3.- Small nerve cell area of Fig. 1 enlarged $(\times 300)$. Note regularity and roundness of cells.

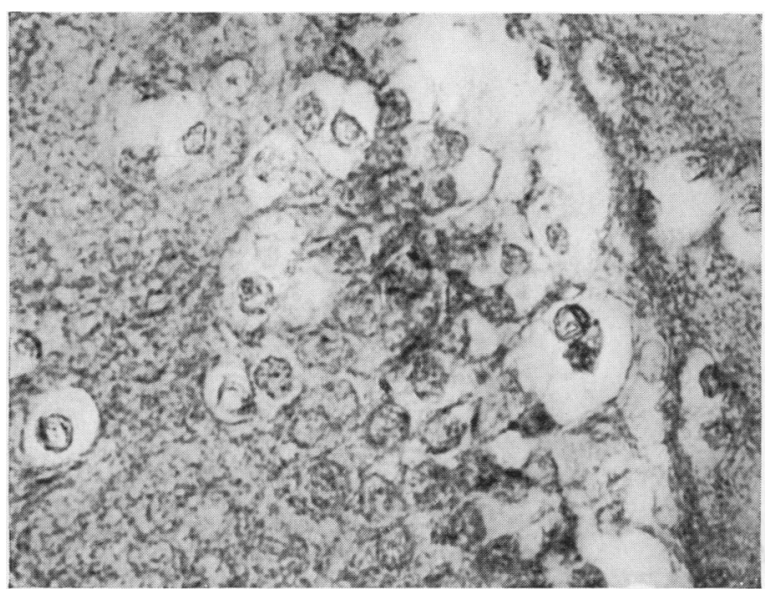

Fig. 7.- Small nerve cell area of Fig. 5 enlarged $(\times 300)$. Note shrinkage and irregularity of cells.

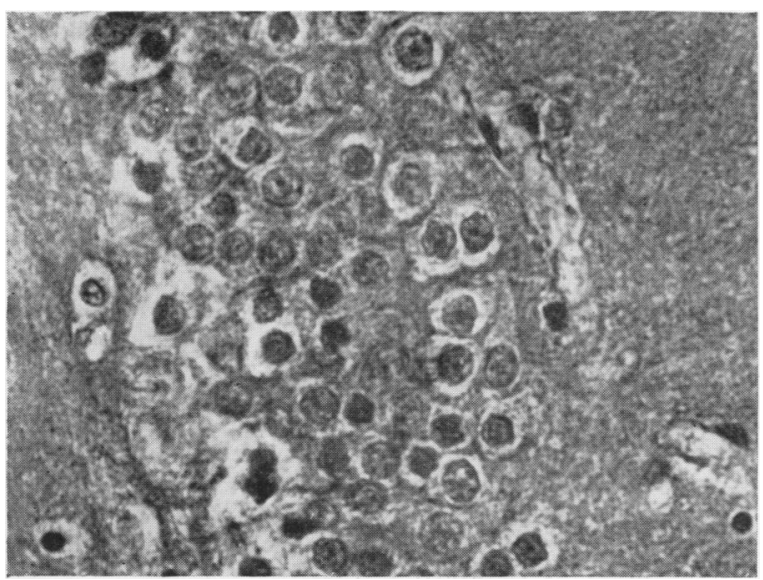

Fig. 11.- Small nerve cell area of Fig. 9 enlarged $(\times 300)$. Note shrinkage of cells and increase in staining.

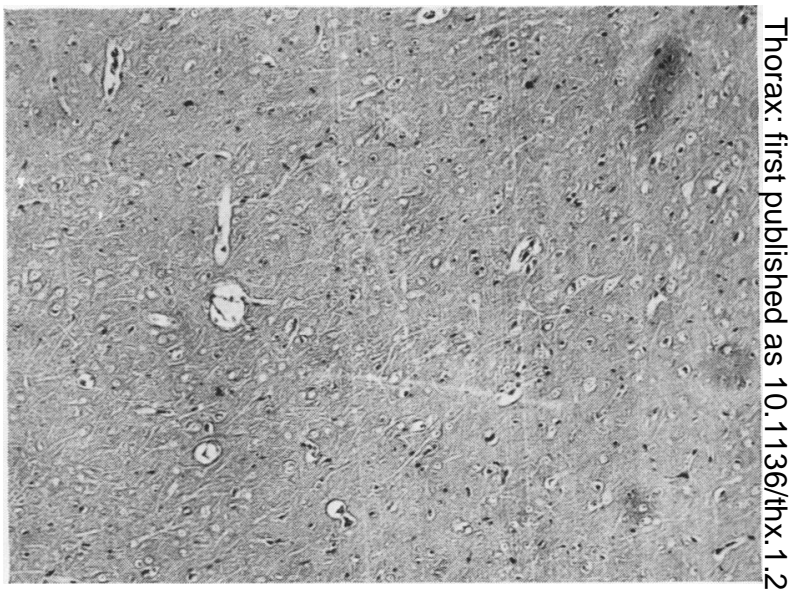

FIG. 4.-Cerebral cortex of normal rat brain $(\times 60)$.

옥

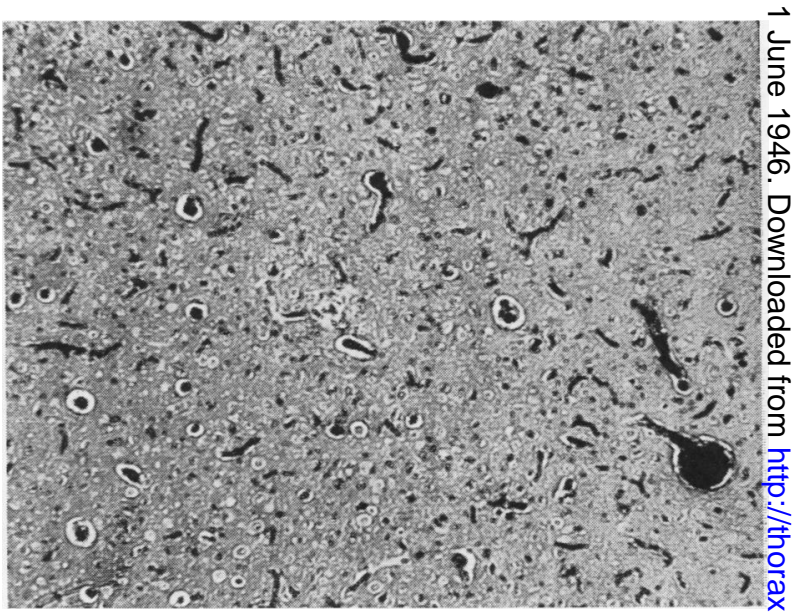

Fig. 8.-Cerebral cortex of anoxic rat which died in chamber $(\times 60)$. Note increase and dilatation of capillaries.

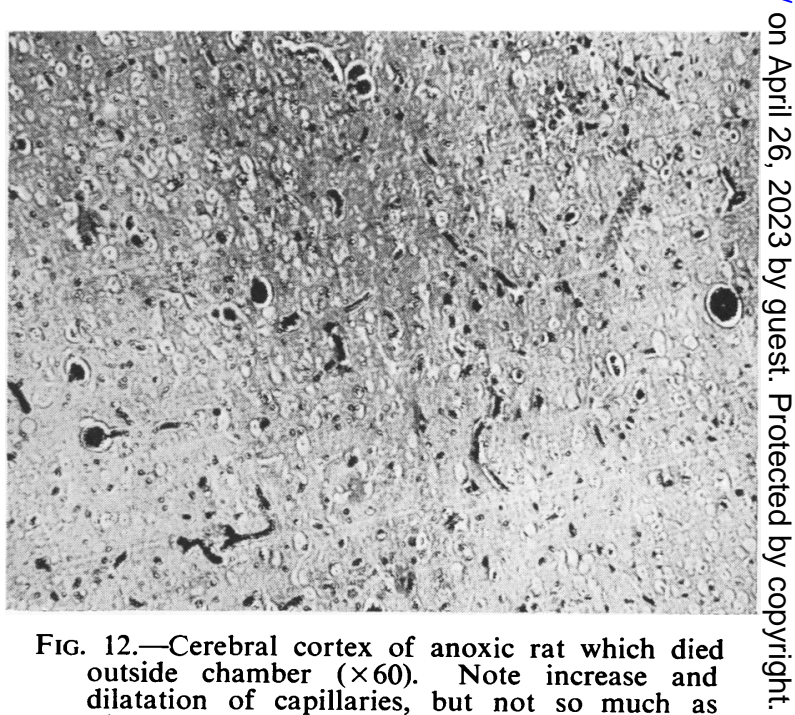
Fig. 8. 


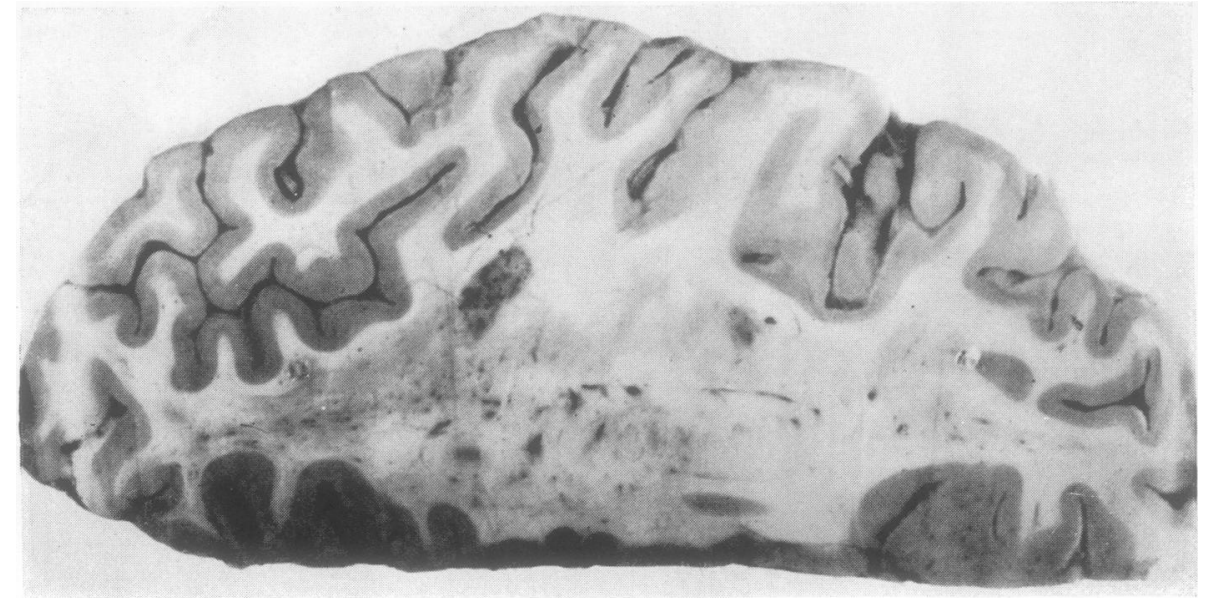

FIG. 13.-Case II. Cerebral slice. Note area of haemorrhage.

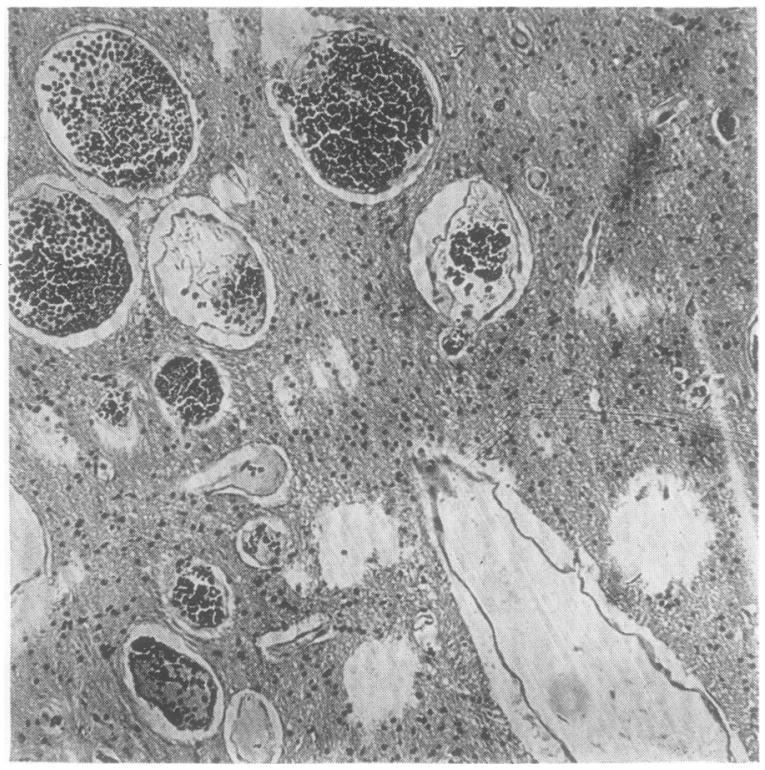

FIG. 14.-Section from area of haemorrhage from Case II $(\times 60)$. Note enormous dilatation of capillaries with no cellular infiltration.

Fig. 15.-Case III. Cerebral slice. Note scattered areas of haemorrhage.

Fig. 16.-Case III. Showing areas of haemorrhage
in corpus striatum.

Fig. 17.-Case III. Cerebellar slice. Note scattered areas of haemorrhage. 


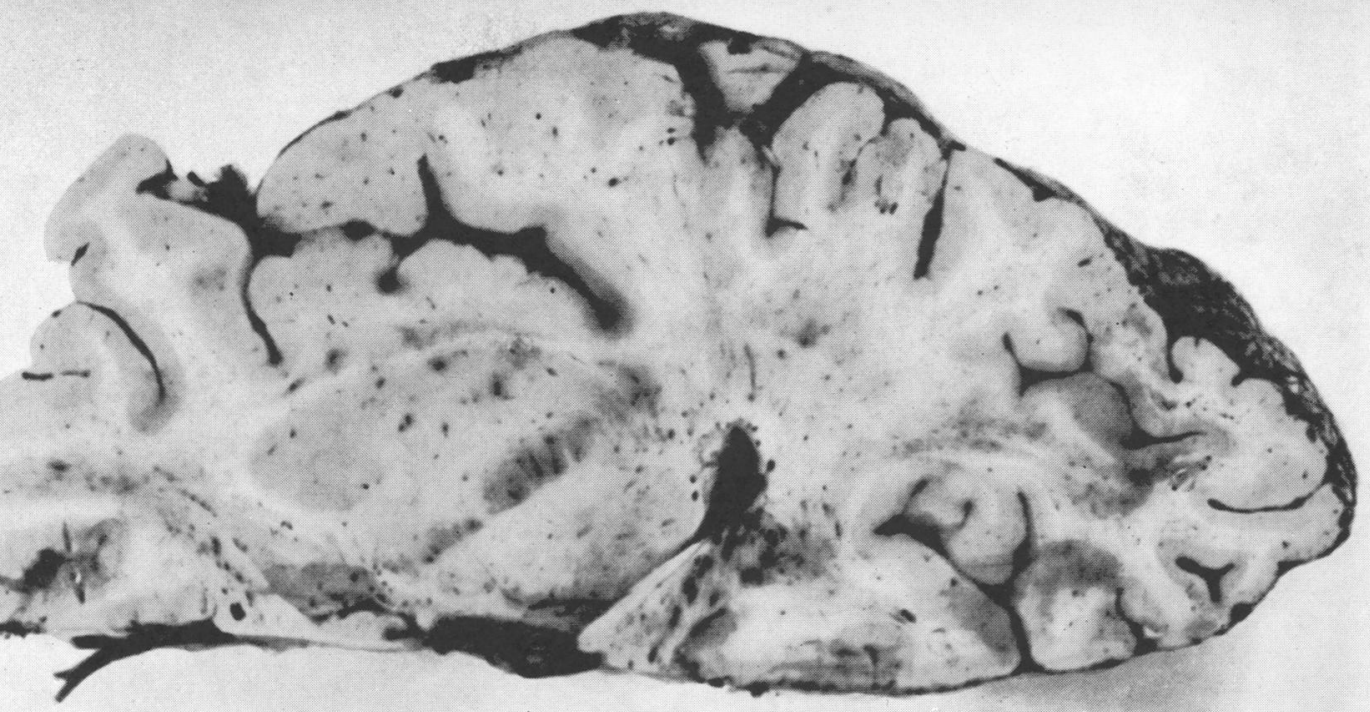

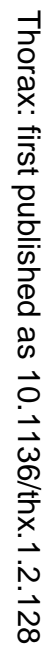

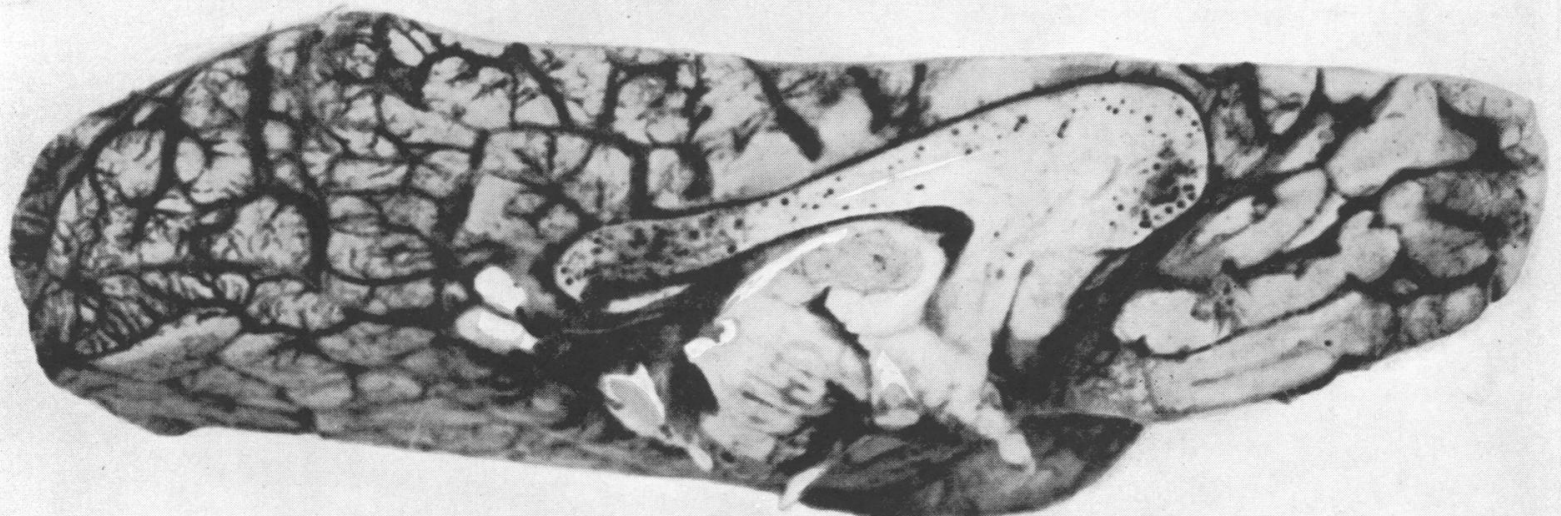

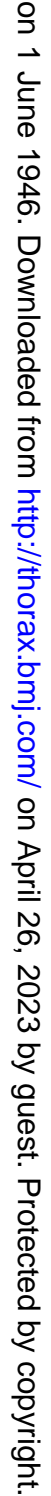

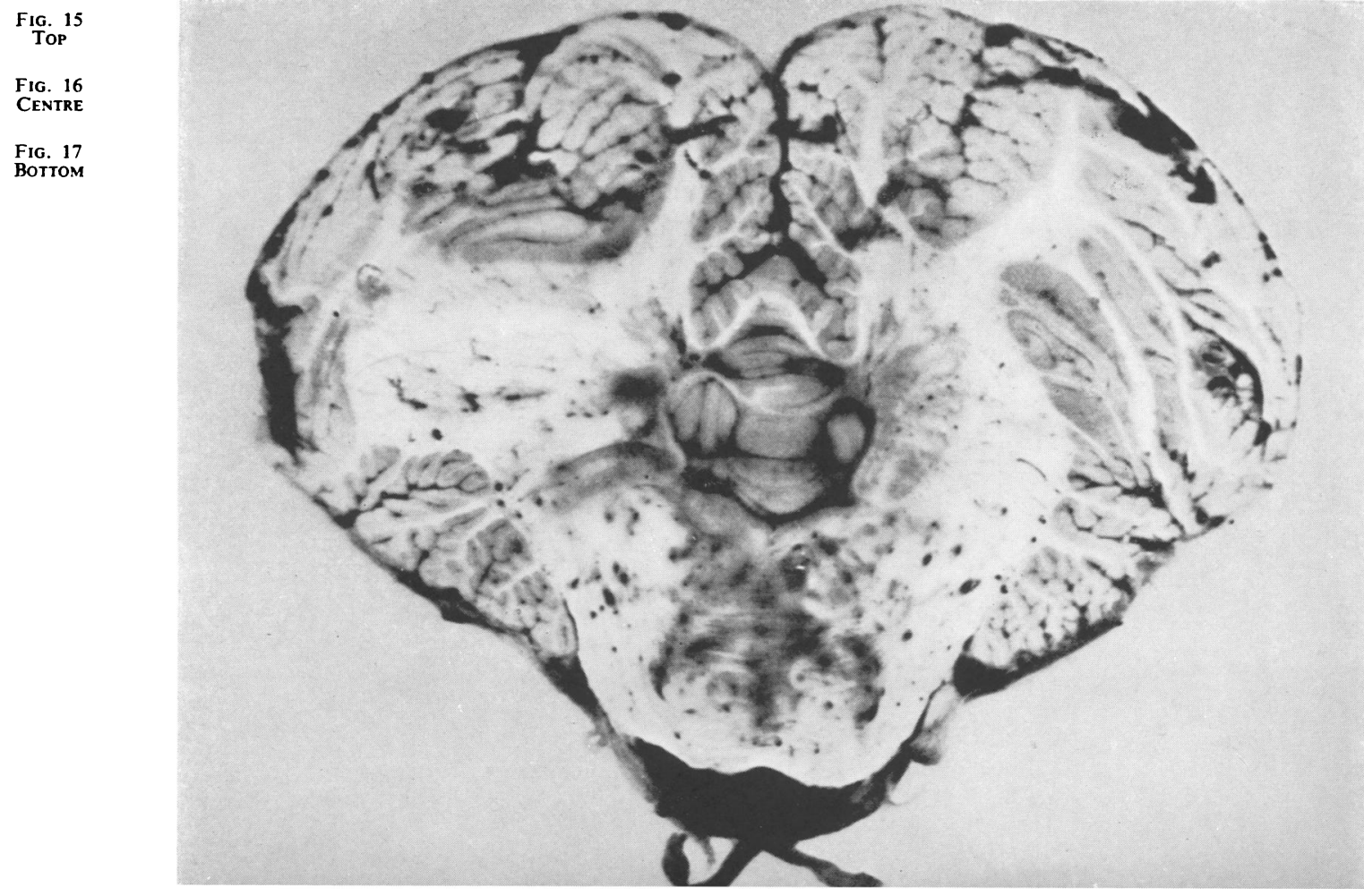




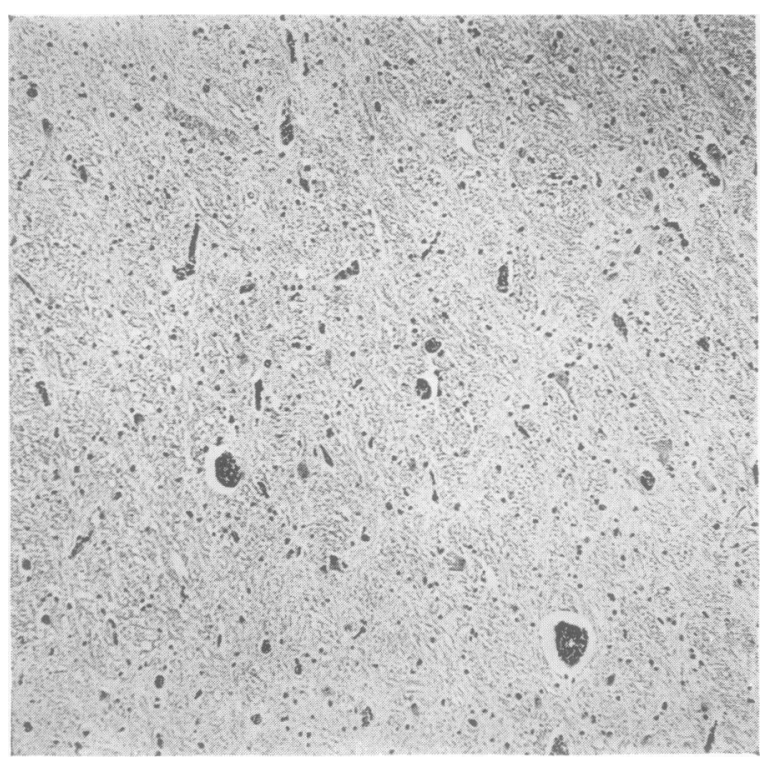

Fig. 18. - Case III. Cerebral cortex. Note dilatation of capillaries and relative lack of nerve cells $(\times 60)$.

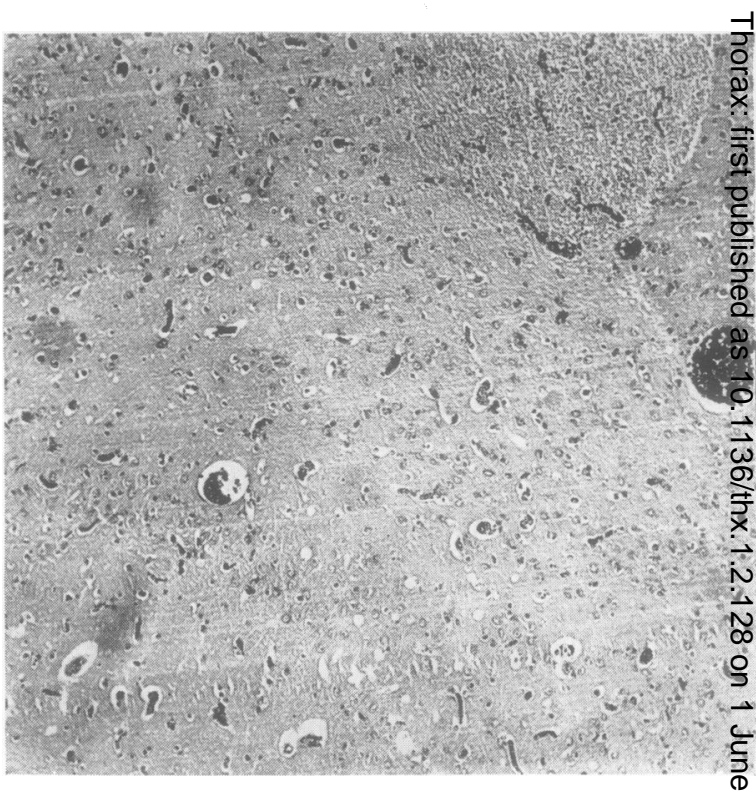

FIG. 19.-Cerebral cortex of anoxic rat brain $(\times 60) . \overrightarrow{0}$ Compare with Fig. 18

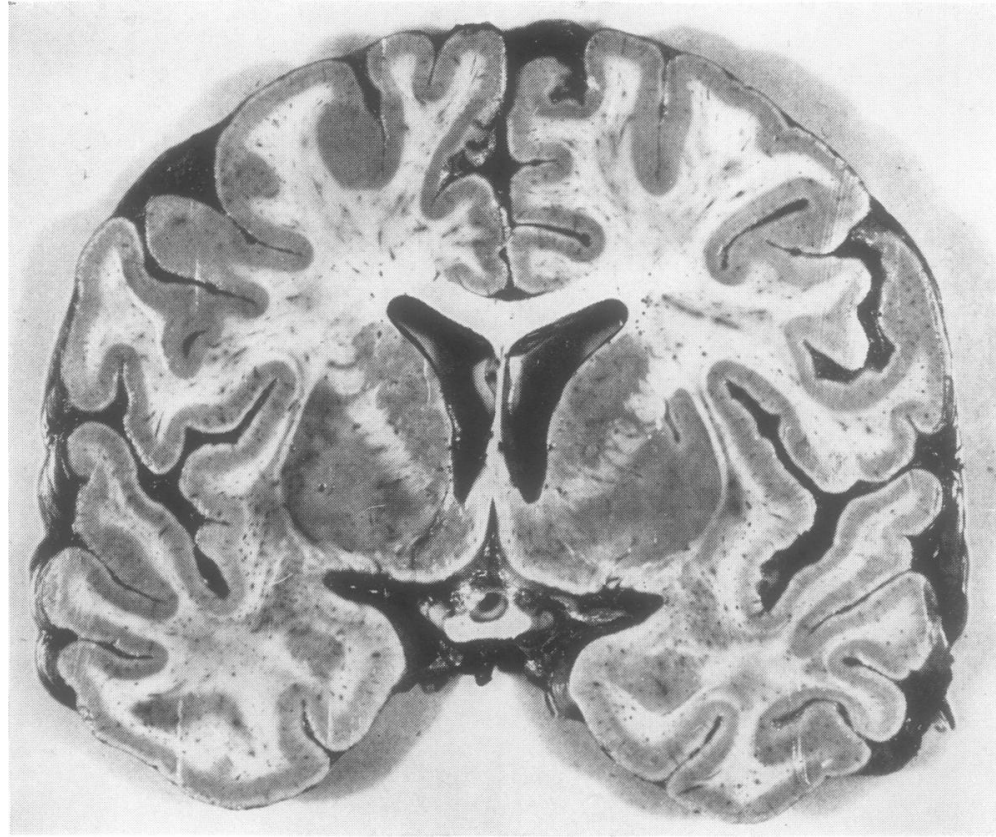

Fig. 20.-Case V. Cross section of cerebral hemispheres. Note ventricular dilatation and widening of sulci. Also scattered areas of haemorrhage. 
Comment: Here the anoxia was largely histotoxic. The combination of omnopon, pentothal, ether, and chloroform might without any other factors have produced respiratory arrest in a man of this age. But in this case the small endotracheal tube, coupled with partial rebreathing, kept the alveolar carbon dioxide at a high level, so stimulating the anoxic respiratory centre. On return to the ward this excess of carbon dioxide was eliminated with subsequent failure of respiration, due to overdosage with drugs. The ensuing anoxic anoxia was the final factor in this old patient in producing the cerebral damage.

CASE III (Female, aged 18). Previously fit, she was operated upon for an adenoma of the thyroid under endotracheal cyclopropane anaesthesia. Operation and return to full consciousness were without incident. Six hours after operation a slight stridor developed and the patient complained of difficulty in breathing. Four hours later she was cyanosed and very dyspnoeic. Oxygen was given somewhat ineffectually with double nasal catheters and the condition soon worsened, until fourteen hours later (i.e., twenty-four hours after the original operation), she was practically unconscious, being very restless, distressed and incoherent. An endotracheal tube was then passed and continuous oxygen given through it. Immediately her colour returned to normal and her general condition improved. Her pulse dropped from 130 to 90 , but mentally her condition did not return to normal. She was confused and emotionally unstable. The following day her condition was unchanged and the tube was removed, but was immediately replaced, as the signs of obstruction at once made themselves manifest, and the oxygen was continued. That evening her mental condition was much worse and by morning she was comatose. Her pulse and temperature both started to rise, and by evening ( $3 \frac{1}{2}$ days after operation) she was deeply unconscious with a pulse rate of 160 and a temperature of $103^{\circ} \mathrm{F}$. She died early the following morning.

Post Mortem: The brain showed areas of haemorrhage scattered throughout its substance, particularly in the white matter, similar to those found in Case II, but much more widespread (Figs. 15 to 17). Microscopically there was much dilatation of capillaries and destruction of cortical nerve cells. This was very similar to that seen in the brains of the anoxic experimental rats (Figs. 18 to 19 ).

Comment: The damage in this case was done during the fourteen hours of partial asphyxia, and nothing could repair it. The gross damage in the brain was so widespread that it is surprising that it produced so few signs.

CASE IV (Male, aged 47). Patient was admitted to hospital following exposure to coal gas. When first seen he was bright red in colour, pulse rate 90 , blood pressure $70 / 50$, and respirations almost imperceptible. Artificial respiration with oxygen and 5 per cent carbon dioxide was started immediately, and a lumbar puncture was done, the pressure being $200 \mathrm{~mm}$. water, and cerebrospinal fluid was allowed to flow slowly out, until the pressure fell to $140 \mathrm{~mm}$. At the same time, a transfusion of one pint of concentrated red cells was given. After an hour respiration started spontaneously when the carbon dioxideoxygen mixture was replaced by oxygen. After six hours the man showed signs of returning consciousness, and oxygen was stopped after nine hours.

The next day he showed all the signs of complete decerebration. He sat propped up in bed with his eyes closed and a mask-like expression on his face, and showed no signs of intelligence whatsoever. He would drink and swallow, if anything were placed in his mouth, and exhibited a perfect physiological "rage reaction"; when pinched he snarled, his face contorted with rage and he moved his limbs feebly, but this movement bore no relation to the site of the painful stimulus. Normally the limbs remained in a state of 
flexion, the muscles being hypertonic. The plantar response was similar to that found in Case II, being a mass withdrawal. The other systems of the body showed no abnormality. He lived, or rather existed, in this state with no recovery whatsoever for a further seven days, finally dying with hyperthermia.

Comment : Unfortunately no post-mortem examination was possible. Had it been, one would have undoubtedly found evidence of cerebral destruction, due to the anaemic anoxia caused by the carbon monoxide, which had not been so severe as to cause immediate death.

CASE V (Female, aged 32). Operated upon for recurrent appendicitis. Anaesthesia was induced with pentothal $0.5 \mathrm{~g}$. and then nitrous oxide and oxygen, followed by a spinal (1.5 c.cm. of heavy nupercaine). The operation had almost been completed when a sudden cardiac failure was reported. Ordinary resuscitation and intubation having failed, cardiac massage was started. After seven minutes the heart commenced to beat, and within a short time was regular and strong in rhythm, the rate being about 90 per minute.

Ten hours after operation the patient showed a modified decerebrate rigidity very similar to that found in Case IV. The plantar response was a mass withdrawal and her face was mask-like and expressionless. She remained in this state for $\mathbf{2 6}$ days, finally dying of pneumonia and pyelonephritis.

Post Mortem: The brain showed a moderate generalized ventricular dilatation with notable widening of the sulci. The thickness of the cortex was diminished and the basal ganglia showed irregular areas of pallor, the largest being in the right corpus striatum (Fig. 20).

Microscopically the whole brain showed extensive nerve cell damage, the changes being particularly marked in the basal ganglia. In the area of the cornu ammonis there was a great loss of pyramidal cells, similar to that found in the experimental rats.

Comment : The anoxia in this case was severe enough, due to the combination of the partial respiratory paralysis of the spinal anaesthetic and the nitrous oxide, to produce cardiac arrest. The circulatory arrest for seven minutes caused widespread brain damage, which was irreversible.*

CASE VI (Male, aged 9). Operated upon for bilateral bronchiectasis. Half way through the operation of right lower lobectomy under endotracheal nitrous oxide-oxygen ether, preceded by chloroform, the air-way became completely obstructed with muco-pus which could not be cleared by suction through the tube. Immediate bronchoscopy was done and the airway cleared, but by this time the heart had stopped and so was massaged. Nikethamine was injected into the heart and then adrenalin. The heart commenced to beat again almost immediately, and after a few minutes resumed normal rhythm. The period of cardiac arrest was at least one minute and the anoxaemia from respiratory obstruction lasted approximately five minutes. Heart-beat and respiration having been re-established, the operation was rapidly completed. Throughout this time, the patient was slightly cyanosed.

The immediate post-operative period was stormy, due to surgical emphysema and much accumulation of secretions. During this time the child appeared dazed but otherwise normal. Three days after the operation he appeared to be blind and mentally abnormal. The following day he became very hysterical and his speech was slurred. He kept his eyes closed but would open them if asked, although he appeared to see nothing.

* This case was reported in full by Howkins, J., McLaughlin, C. R., and Daniel, P. (1946) Lancet, 1, 488. 
His pupils were dilated but responded to light, and his optic disks showed temporal pallor and blurring of outline on the nasal side. He was completely spastic and incontinent. This state persisted for several days. Gradually his condition improved, and three weeks after the operation he appeared more normal mentally; his speech was more distinct and his limbs, although still ataxic, were capable of some voluntary movement.

Eight months after operation he was walking without assistance although he was ataxic and the spasticity of the limbs persisted. Mentally, he was rational, but seemed to have a very poor memory. His face was practically devoid of expression.

Comment : Here the severe anoxia was brief, but a mild degree continued throughout the operation. This was enough to produce some cerebral and cerebellar damage. There appears to be some recovery, but some damage still persists as shown by the ataxia. At this stage it is difficult to assess the cortical damage, but the poor memory is probably significant and if it persists will lead to impairment of the child's intelligence.

CASE VII (Male, aged 64). Heavy build but otherwise fit. Operated upon for carcinoma of the lung. During the operation of left pneumonectomy under controlled respiration cyclopropane anaesthesia the blood pressure fell suddenly to 50/30 for a period of 15 minutes, although it rose again to $100 / 75$ at the close of the operation. Recovery was fairly rapid, but the patient was very restless. The next day he was mentally confused and appeared completely drunk. The following day the confusion had disappeared, being replaced by a state of euphoria. This was accompanied by marked slurring and scanning of speech. All neurological investigations were negative, including a Wassermann reaction. Three months later the patient was still euphoric with an ataxic gait and slurred speech. There is a little doubt about the euphoria, as the physician in charge of the patient before operation was unable to detect any difference post-operatively.

Comment: A possible causative factor here is stagnant anoxia caused by the low blood pressure for 15 minutes during the normal anaesthesia. There may, too, have been an anoxic element post-operatively due to the effects of the pneumonectomy itself. These, in an old patient, were sufficient to cause psychotic changes and some cerebellar damage.

CASE VIII (Male, aged 5). Operated upon for bilateral bronchiectasis. The operation of left lower lobectomy under endotracheal nitrous oxide-oxygen-ether anaesthesia, preceded by chloroform, was completed without incident, and the condition and colour on leaving the table were good. He was at once placed in an oxygen tent, but within a few hours he became very restless and pale. Phenobarbitone was given to control the restlessness. The next day he was still unconscious, and even on the third day he was drowsy and hardly responded to stimulation. It was not until the fifth day that his condition ceased to give rise to anxiety. He was discharged from hospital after one month, apparently well. defective.

Three years later, his general condition was improved, but he was now a mental

Comment: The evidence in this case is not conclusive as no real assessment of the child's intelligence was made prior to operation; in fact, the ward sister thought that the child was somewhat mentally defective before operation. But it is probable, from the evidence of the delayed recovery, that some cortical damage occurred which only became manifest later. 


\section{Discussion}

From this experimental and clinical evidence it appears that prolonged anoxia can cause serious brain damage, varying from death in a few hours to mental changes in an otherwise normal brain. Also, it seems that clinically a combination of different types of anoxia is more dangerous than any one particular type.

The degree of damage is largely dependent upon the severity and duration of the anoxia, but not wholly so. There is the factor of inherent resistance of the brain to anoxia ; Fazekas and Himwich (1943) have shown that this decreases with age, so that old subjects have relatively little cerebral reserve. This probably accounted for the cortical damage that occurred in Case VII, whose anoxia was very mild. From the animal experiments, too, it seems that this reserve can be lowered by repeated or continuous exposures to low oxygen tension.

How is it possible to diagnose these mild degrees of oxygen lack clinically? In gross anoxia the physical signs are obvious, but in the minor degrees they may not be so. There are, however, a number of signs which individually may not denote oxygen want, but together almost invariably do. These signs are:

(1) Increased respiratory rate, possibly accompanied by dyspnoea. One of the earliest indications of dyspnoea is the bringing into use of the accessory muscles of respiration, particularly the alae nasi; this sign is remarkably constant, but in its mildest form may only show itself as a very slight expansion of the nostrils on inspiration.

(2) Increased heart rate accompanied by an initial increase in blood pressure which is rapidly followed by a fall. If it is suspected that a raised pulse rate is due to anoxic anoxia this can be proved by making the patient inhale oxygen for two minutes while at complete rest. If the pulse rate drops, even a few beats, the diagnosis is confirmed. This test is perhaps most useful when one wishes to give a patient continuous oxygen-air mixtures. A suitable mixture is that which produces a lowering of the pulse rate with the minimum amount of oxygen.

(3) Restlessness and delirium. The reason for this restlessness is not fully understood, but it is probably due partly to the immediate effect of anoxia on the higher brain centres, and also to dyspnoea.

This clinical picture is best seen after an operation which has not proceeded smoothly from an anaesthetic point of view, and has been followed by a period of respiratory depression. Recovery is delayed and there is mental confusion. All too often these patients are stated to be "restless" or "hysterical," whereas their mental state is attributable to the anoxia during or after the operation. This type of syndrome is commonest in the age groups 14 to 25 and over 60 . In the former group ultimate recovery seems to be the rule, but in the over 60 group the reverse is true, and there may be psychotic 
changes. These are not infrequently regarded as due to premature senility or to cerebral metastases of malignant disease.

(4) Excessive sweating and pallor.

In thoracic surgery particularly, the dangers of anoxia cannot be overemphasized, and failure to appreciate these dangers has led to many disasters in the past. It is still not uncommon for the aged patient to die suddenly a few days after an extensive thoracotomy, death being probably due to prolonged mild anoxia associated with a low resistance of the brain to anoxia.

The gross disturbances of respiration that occur in thoracic surgery are all capable of producing mild degrees of anoxia, and these disturbances continue for some days after operation, not, as is commonly supposed, only while the thorax is opened.

During this period measures must be instituted to combat this anoxia. Treatment must be prophylactic, as when the effects of anoxia become apparent a vicious circle will have been already started and treatment will be less effective. As the age of the patient increases, so the margin of safety diminishes; and it is in the older patients particularly that anoxia should be reduced to the minimum.

\section{SUMMARY}

The effects of prolonged mild anoxia on the central nervous system are described. Experiments on rats show that 9 per cent oxygen for a period of 60 to 70 hours produces signs of neurological damage and often death. The experimental evidence suggests that the effects of mild anoxia are cumulative.

Eight human cases are reported in which anoxia was responsible for either death or permanent neurological damage.

The signs of mild oxygen want are increased respiratory rate and dyspnoea, increased heart rate, restlessness and delirium, and sweating and pallor.

In thoracic surgery anoxia is especially likely to occur, as respiration is disturbed, and the greatest care must be taken to reduce this anoxia to the minimum.

\section{ACKNOWLEDGEMENTS}

I am indebted to Dr. Dorothy H. Strangeways, of University College, Cardiff, who has been responsible for the histological part of the investigations on this subject.

I wish to thank Dr. Gethro Gough, pathologist to the Royal Infirmary, Cardiff, for his help in preparing the post mortem and pathological reports of the human cases.

\section{REFERENCES}

Brown, W. E., Lucas, G. H. W., and Henderson, V. E. (1927). J. Pharmacol and Exp. Therap., 31, 269 .

Courville, C. B. (1938). Ann. Surg., 107, 371.

Dellaporta, A. N. (1939). Beitr. Path. Anat., 102, 268.

Fazekas, J. F., and Himwich, H. E. (1943). Am. J. Physiol., 139, 366. 
Gildea, E. F., and Cobb, S. (1930). Arch. Neurol. and Psychiat., 23, 876

Helwig, F. C. (1937). Southern Med. J., 30, 531.

Lenahan, N. E. (1943). Anaesthesiology, 4, 543.

Merk, R. (1940). Arch. Psychiat., Berl., 111, 160.

O'Brien, J. D., and Steegmann, A. T. (1938). Ann. Surg., 107, 486.

Oster, R. H., Toman, J. E. P., and Smith, D. C. (1944). Am. J. Physiol., 141, 410.

Steegmann, A. T. (1939). Arch. Neurol. and Psychiat., 41, 955.

Suggs, W. D. (1943). Virginia Med. Monthly, 70, 513.

Thorner, M. W., and Lewy, F. H. (1940). J.A.M.A., 115, 1595.

Yaskin, J. C., and Thorner, M. W. (1943). Trans. Am. Neurol. Ass., 1943, 69, 88. 